\title{
Tau filament formation and associative memory deficit in aged mice expressing mutant (R406W) human tau
}

\author{
Yoshitaka Tatebayashi*†, Tomohiro Miyasaka*, De-Hua Chui*, Takumi Akagi ${ }^{\ddagger}$, Ken-ichi Mishima\$, Katsunori Iwasaki\$, \\ Michihiro Fujiwara§, Kentaro Tanemura*, Miyuki Murayama*, Koichi Ishiguro", Emmanuel Planel*, Shinji Sato*, \\ Tsutomu Hashikawa ${ }^{\ddagger}$, and Akihiko Takashima*\|

\begin{abstract}
*Laboratory for Alzheimer's Disease and ${ }^{\ddagger}$ Neural Architecture, Brain Science Institute, Institute of Physical and Chemical Research (RIKEN), 2-1 Hirosawa, Wako-shi, Saitama 351-0198, Japan; §Department of Physiology and Pharmacology, Faculty of Pharmaceutical Sciences, Fukuoka University, 8-19-1 Nanakuma, Jonan-Ku, Fukuoka 814-0180, Japan; and "Mitsubishi Kasei Institute of Life Sciences, 11 Minamiooya, Machida,
\end{abstract} \\ Tokyo 194-8511, Japan
}

Edited by Eric M. Shooter, Stanford University School of Medicine, Stanford, CA, and approved August 19, 2002 (received for review April 8, 2002)

The R406W tau mutation found in frontotemporal dementia and parkinsonism linked to chromosome 17 (FTDP-17) causes a hereditary tauopathy clinically resembling Alzheimer's disease. Expression of modest levels of the longest human tau isoform with this mutation under the control of the $\alpha$-calcium-calmodulindependent kinase-II promoter in transgenic $(\mathrm{Tg})$ mice resulted in the development of congophilic hyperphosphorylated tau inclusions in forebrain neurons. These inclusions appeared as early as 18 months of age. As with human cases, tau inclusions were composed of both mutant and endogenous wild-type tau, and were associated with microtubule disruption and flame-shaped transformations of the affected neurons. Straight tau filaments were recovered from Sarkosyl-insoluble fractions from only the aged Tg brains. Behaviorally, aged Tg mice had associative memory impairment without obvious sensorimotor deficits. Therefore, these mice that exhibit a phenotype mimicking R406W FTDP-17 provide an animal model for investigating the adverse properties associated with this mutation, which might potentially recapitulate some etiological events in Alzheimer's disease.

$\mathbf{N}$ eurofibrillary tangles (NFTs) composed of abnormally hyperphosphorylated microtubule-associated protein tau are prominent in certain types of neurodegenerative diseases. Examples of such tauopathies include Alzheimer's disease (AD), progressive supranuclear palsy, corticobasal degeneration, Pick's disease, and frontotemporal dementia and parkinsonism linked to chromosome 17 (FTDP-17) (for review, see refs. 1-5). The discovery of multiple tau gene mutations in FTDP-17 provides evidence that tau abnormalities alone can cause neurodegenerative diseases (6-9).

In FTDP-17, mutations within exon 10 or its $5^{\prime}$ splice regulatory region alter the ratio of tau isoforms incorporated into tangles and result in filamentous tau inclusions resembling those in primary tauopathies, including progressive supranuclear palsy, corticobasal degeneration, and Pick's disease (1). Generally, these mutations affect the alternative splicing of exon 10 and consequently alter the relative proportion of four-repeat (4R) to three-repeat (3R) tau expressed $(6,8,10,11)$. The exonic mutation P301L, however, does not affect this proportion, but instead appears to promote the self-assembly of mutant tau into filaments, resulting in the selective incorporation of $4 \mathrm{R}$ (mutant) tau into tangles $(12,13)$. A similar tendency of mutant tau to self-assemble into filaments appeared to be observed in transgenic (Tg) mice expressing P301L human tau that showed an age- and gene-dose-dependent accumulation of NFTs in the brain and spinal cord $(14,15)$.

In contrast, missense mutations affecting constitutively expressed exons affect all six tau isoforms and result in NFTs similar to those present in secondary tauopathies, such as AD (1). For example, patients with the R406W or V337M tau mutation have paired helical and/or straight tau filaments that consist of all six tau isoforms; these filaments are indistinguishable from those seen in $\mathrm{AD}$ brains (4, 16-18). Immunocytochemical and biochemical analysis of brains from R406W patients by using antibodies specific for R406W tau revealed that both mutant and wild-type tau were deposited in NFTs and recovered from insoluble fractions (19). Furthermore, the R406W (but not V337M) mutation causes AD-like clinical symptoms (e.g., gradual progression of memory loss and personality change) in humans mainly without amyloid $\beta$ deposition (17), suggesting a possibility that some of the adverse properties associated with R406W mutation might recapitulate some early clinicopathological events in AD.

To determine whether the R406W tau mutation is associated with a similar phenotype in mice, we expressed modest levels of the longest human tau isoform with this mutation in $\mathrm{Tg}$ mice. Although Lim et al. (20) reported mice expressing human tau with triple FTDP-17 mutations (G272V, P301L, and R406W) that showed accelerated NFT formation, no such mice with single R406W tau mutation have been reported. We used the $\alpha$-calcium-calmodulin-dependent kinase II (CaMK-II) promoter (21) for the expression of human tau because earlier studies indicated that the expression of CaMK-II mRNA starts postnatally, mainly in the forebrain neurons (22). These areas are known to play central roles in learning and memory and overlap with the affected areas in R406W patients (17). We show here that these Tg mice develop congophilic hyperphosphorylated tau inclusions as early as 18 months of age. Furthermore, fear conditioning, a kind of associative memory, was impaired in these mice; no obvious sensorimotor deficits were apparent. These mice, therefore, display a disease phenotype that mimics R406W FTDP-17 and, partially, AD, and provide an animal model for investigating the mechanisms of neurofibrillary formation, a characteristic pathological event in $\mathrm{AD}$.

\section{Materials and Methods}

Generation of Tg Mice Expressing R406W Human Tau. The generation of Tg mouse lines expressing R406W human tau was performed as described $(23,24)$, except that the CaMK-II promoter $(21)$ was used for the expression. A cDNA construct of R406W human tau containing myc (EQKLISEEDL) and FLAG (DYKDDDDK) tags at the N- and C-terminal ends, respectively, was inserted into the CaMK-II chain expression vector at the XhoI and NotI

This paper was submitted directly (Track II) to the PNAS office.

Abbreviations: NFTs, neurofibrillary tangles; AD, Alzheimer's disease; FTDP-17, frontotemporal dementia and parkinsonism linked to chromosome-17; $4 R$, four-repeat; 3R, threerepeat; Tg, transgenic; CaMK-II, $\alpha$-calcium-calmodulin-dependent kinase II.

${ }^{\dagger}$ Y.T. and T.M. contributed equally to this work.

"To whom correspondence should be addressed. E-mail: kenneth@brain.riken.go.jp. 
sites. A 4.3-kb BglII-NaeI fragment containing the CaMK-II promoter, R406W human tau cDNA, and a $3^{\prime}$ untranslated sequence was used as the transgene to create the $\mathrm{Tg}$ mice on a B6SJL background. On the basis of Southern and Western blot analyses, four founder lines ( $\mathrm{Tg} 748, \mathrm{Tg} 502, \mathrm{Tg} 492$, and $\mathrm{Tg} 483$ ) were expanded with the four highest transgene expression levels $(\operatorname{Tg} 748>\operatorname{Tg} 502>\operatorname{Tg} 492>\operatorname{Tg} 483$; data not shown $)$.

Antibodies. The following antibodies were used: mouse monoclonal anti-myc (clone 9E10; Babco, Richmond, CA); anti- $\alpha$ tubulin (DM1A, Sigma); anti-ubiquitin (P4D1, Santa Cruz Biotechnology); phosphorylation-independent anti-tau H150 (for the recognition of human tau; Santa Cruz Biotechnology); rabbit polyclonal anti-tau JM (25); phosphorylation-dependent mouse monoclonal anti-tau AT180 (Innogenetics Zwijndrecht, Belgium), which recognizes tau phosphorylated at Ser-231 and Thr-235, Tau-1 (Chemicon), which recognizes tau dephosphorylated at Ser-195, -198, -199 and -202; phosphorylationdependent rabbit polyclonal anti-tau antibodies PS199, PT205, PS396, PS404, PS413, and PS422 (25), which recognize tau phosphorylated at each corresponding site; mouse monoclonal anti-tau antibody Alz50 (26), which recognizes the conformational epitope of paired helical filaments (the generous gift of $\mathrm{P}$. Davies, Albert Einstein College of Medicine, Bronx, NY); and rabbit polyclonal antibodies generated against mutant R406W (AW406) and the corresponding wild-type tau (AR406) (the generous gift of Y. Ihara, University of Tokyo) (19).

Brain Fractions. Brains were extracted after euthanasia with anesthesia and homogenized in Tris-buffered saline [TBS; 10 $\mathrm{mM}$ Tris/150 mM NaCl ( $\mathrm{pH} 7.4)$ ] containing protease inhibitors [1 $\mu \mathrm{g} / \mathrm{ml}$ antipine, $5 \mu \mathrm{g} / \mathrm{ml}$ pepstatin, $5 \mu \mathrm{g} / \mathrm{ml}$ leupeptin, 2 $\mu \mathrm{g} / \mathrm{ml}$ aprotinin, and $0.5 \mu \mathrm{M} 4$-(2-aminoethyl)benzenesulfonyl fluoride hydrochloride] and phosphatase inhibitors (1 mM NaF, $0.4 \mu \mathrm{M} \mathrm{Na}_{3} \mathrm{VO}_{4}$, and $0.5 \mu \mathrm{M}$ okadaic acid). After centrifugation at $100,000 \times g$ for $15 \mathrm{~min}$, the supernatant was collected. If needed, precipitation was carried out by adding saturated ammonium sulfate to TBS-soluble fractions. The resulting precipitate was resuspended in dephosphorylation buffer $(50 \mathrm{mM}$ Tris, $\mathrm{pH} 8.3$ ) containing protease inhibitors and incubated with 2 units/ml of bacterial alkaline phosphatase (Sigma, type III) at $37^{\circ} \mathrm{C}$ for $2 \mathrm{~h}$. Sarkosyl-insoluble, paired helical filament-enriched fractions were prepared from TBS-insoluble pellets according to the procedure developed by Greenberg and Davies (27). For Western blotting analysis, the ECL system (Amersham Pharmacia) was used with a computer-linked LAS-1000 Bio-Imaging Analyzer (Fuji) using the program IMAGE GAUGE 3.0 (Fuji).

Immunohistochemical Procedures. After an intracardial perfusion of buffered $10 \%$ formalin, brains were postfixed in the same fixative for $16 \mathrm{~h}$ and embedded in paraffin. Deparaffinized sections $(4-6 \mu \mathrm{m})$ were treated in Target Retrieval Solution (Dako) for $20 \mathrm{~min}$ at $80^{\circ} \mathrm{C}$, blocked in $0.1 \% \mathrm{BSA} / \mathrm{TBS}$ and incubated with primary antibodies in $0.1 \% \mathrm{BSA} / \mathrm{TBS}$ overnight at $4^{\circ} \mathrm{C}$. A Radiance $2000 \mathrm{KR} 3$ confocal microscope (Bio-Rad) was used for the observations. Thioflavin S, Congo red, and Gallyas silver staining were performed as described (23, 24). For some experiments, frozen sections were also prepared. After postfixation, brains were cryoprotected with a $30 \%$ sucrose solution in $0.1 \mathrm{M}$ sodium phosphate buffer, $\mathrm{pH} 7.4$, embedded in Tissue-Tec (Miles), and frozen by using liquid nitrogen. Thirty-micrometer-thick sagittal sections were cut with a cryostat (CM 1510, Leica). Free-floating sections were incubated with primary antibodies as above.

Ultrastructural Study. After intracardial perfusion with $25 \mathrm{ml}$ of Ringer's solution, followed by $4 \%$ paraformaldehyde and $0.25 \%$ glutaraldehyde in $0.1 \mathrm{M}$ phosphate buffer, brain slices $(300 \mu \mathrm{m})$ were prepared with a microslicer. Slices were postfixed in $1 \%$ osmium tetroxide, dehydrated in a graded series of ethanol solutions, infiltrated with propylene oxide, and embedded in Araldite. Ultrathin sections were cut with an ultramicrotome, stained with uranyl acetate and lead citrate, and examined with a LEO912AB transmission electron microscope (Zeiss) at $100 \mathrm{kV}$.

Behavioral Tests. Mice (16-23 months old) were housed, four to five per cage, with access to food and water and maintained on a 12-h light/dark cycle (lights on at 6:00 a.m.). All of the behavioral tests were performed as described (28). Motor coordination and balance were tested with the rotarod test, and neuromuscular strength was tested with the pole test. Locomotor activity was measured by using an open-field test. A startle reflex measurement system (MED Associates, St. Albans, VT) was used for the startle response/prepulse inhibition tests. Associative memories were assessed by using contextual and cued fear conditioning. Images were analyzed with the custom applications (IMAGE EP and IMAGE FZ) developed from the public domain NIH IMAGE program (developed by Wayne Rasband, U.S. National Institute of Mental Health, Bethesda).

Statistical Analysis. Statistical analyses were conducted by using STATVIEW (SAS Institute, Cary, NC). Data were analyzed by using two-tailed $t$ tests, two-way ANOVA, or two-way repeatedmeasures ANOVA, unless noted otherwise. Values in tables and graphs are expressed as means \pm SEM.

\section{Results}

An $8.5-\mathrm{kb}$ portion of the CaMK-II promoter containing upstream control regions and the transcriptional initiation site drives expression postnatally in forebrain neurons (21). Western blot analysis revealed that mutant tau expression was greatest in the hippocampus $(17.8 \pm 0.35 \%$ of corresponding endogenous tau levels), followed in decreasing amounts by the neocortex $(17.0 \pm 0.92 \%)$, the olfactory bulb $(14.3 \pm 1.07 \%)$, the striatum $(14.1 \pm 0.60 \%)$, and the thalamus $(7.59 \pm 0.41 \%)$; mutant tau was barely detectable in the midbrain, the cerebellum, and the spinal cord (Fig. 1A, 5 months). The expression patterns were almost the same among the lines. Immunohistochemical analysis with anti-human tau (H-150, Fig. $1 B)$ antibody confirmed this regional specificity; it selectively stained mainly forebrain neurons of the Tg brains (5 months).

We next examined aged $\mathrm{Tg}$ mice ( $>18$ months) for neuropathological changes. Immunostaining with anti-myc antibodies confirmed the selective neuronal expression and accumulation of mutant tau in some of aged Tg brains. We observed occasional intense myc immunoreactivity in neurons from the hippocampus (Fig. 2a), striatum, amygdala, neocortex, and olfactory bulb. We never observed myc-positive cells in the brains of non-Tg littermates. Myc-positive cells were also stained with a series of tau antibodies recognizing phosphorylated (phospho-Ser-199, -202, -396, -404, -413, and -422, and Thr-205; AT180, Fig. $2 c-h$ and data not shown), conformational (Alz50, Fig. 2i), and phosphorylation-independent (JM, Fig. $2 k$ and $o$ ) tau epitopes. The antibodies were also, sometimes, immunoreactive for ubiquitin (Fig. 2j). Some of the tau antibodies (AT180, Fig. 2e) clearly stained the somatodendritic compartments of neurons. In a subset of these neurons, we identified argyrophilic and congophilic inclusions (Fig. $2 m-t$ ).

We next assessed the insolubility of tau in aged Tg brains. In Sarkosyl-insoluble fractions, we often found tau recovered only from aged Tg brains (Fig. $3 A$ ). The proportion of mutant to endogenous tau in Sarkosyl-insoluble fractions (12.2 $\pm 1.29 \%)$ were almost same as that in TBS (Sarkosyl-soluble) fractions $(11.6 \pm 0.49 \%)$, suggesting that the mutant and the wild-type tau were proportionally incorporated into the insoluble fractions in Tg brains. When the Sarkosyl-insoluble materials were subjected 


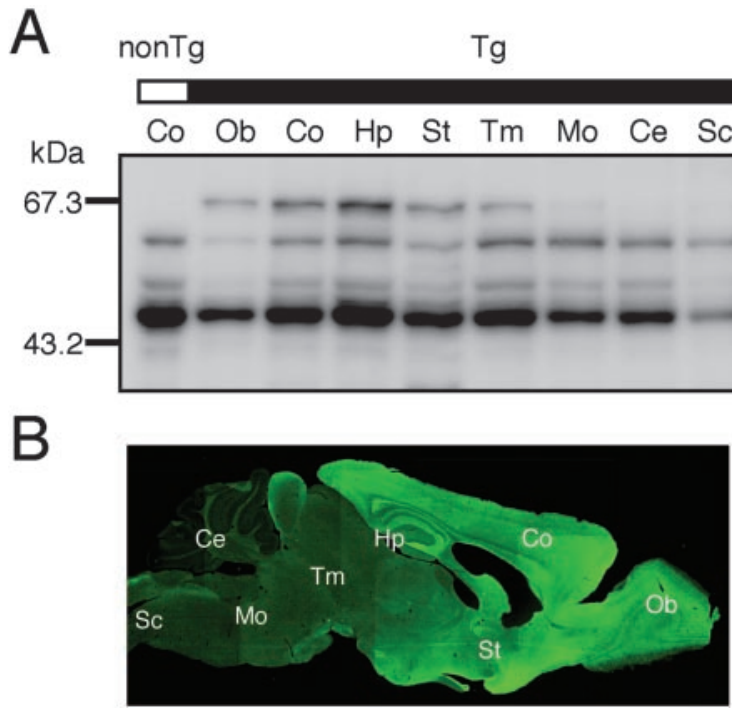

Fig. 1. Expression profile of mutant tau in Tg mice. $(A)$ Western blot analysis of tau expression in Tg mice (5 months). Equal amount of proteins of TBSsoluble fractions from the cortex (Co), olfactory bulb (Ob), hippocampus ( $\mathrm{Hp}$ ), striatum (St), thalamus and midbrain (Tm), medulla oblongata (Mo), cerebellum (Ce), and spinal cord (Sc) were dephosphorylated, subjected to SDS/PAGE, and probed with Tau-1. An arrowhead indicates the position of the mutant tau. (B) A fluorescent photomicrograph of a sagittally sectioned $\mathrm{Tg}$ brain immunostained with the anti-human tau antibody $(\mathrm{H}-150)$. Note that mutant tau is robustly expressed in the forebrain regions, but weakly expressed in the cerebellum and brainstem.

to ultrastructural analysis, we observed bundles of tau filaments that were mainly straight $(\approx 10 \mathrm{~nm}$ in diameter $)$ and immunostained with anti-tau antibody, JM (Fig. $3 B$ ).

To further characterize how endogenous mouse tau is involved in the formation of tau inclusions, we used polyclonal antibodies AR406 and AW406 raised against wild-type and mutant human tau, respectively (19). These antibodies selectively recognized mouse wild-type and mutant human tau, respectively (Fig. 4A). Immunostaining of serial sections with these antibodies revealed that most of the AW406-positive irregularly shaped neurons were also immunoreactive for AR406 (Fig. 4B). Double-labeling with the anti-myc and AR406 antibodies confirmed the simultaneous accumulation of mutant and wild-type tau in the same neurons (data not shown). Because some of these neurons develop congophilic tau inclusions, these data, in combination with the biochemical analysis (Fig. $3 A$ ), indicate that the simultaneous accumulation of mutant and endogenous wild-type tau probably precedes neurofibrillary formation in $\mathrm{Tg}$ brains.

Vulnerable neurons, especially those in CA1 and CA 2 of the hippocampus, were morphologically heterogeneous and transformed into flame-like shapes. We, therefore, studied microtubule structures in the aged $\mathrm{Tg}$ hippocampus. Doubleimmunostaining with anti- $\alpha$-tubulin and AR406 antibodies revealed that the irregularly shaped neurons that were positive for AR406 lacked cytosolic tubulin immunoreactivity, whereas the remaining intact neurons that displayed less AR406 immunoreactivity were tubulin-positive (Fig. $4 C-F$ ). Ultrastructural analysis further revealed that these irregularly shaped neurons were electron-dense, filled with ribosomes, mitochondria, and lipofuscin but were devoid of microtubules (Fig. 4H). We sometimes found bundles of thin filaments in these neurons that were reminiscent of tau filaments found in Sarkosyl-insoluble fractions from the aged Tg brains (Fig. 4I; see also Fig. $3 B$ ). These data suggest that microtubule disruption probably occurs in these irregularly shaped neurons in association with the formation of tau inclusions.
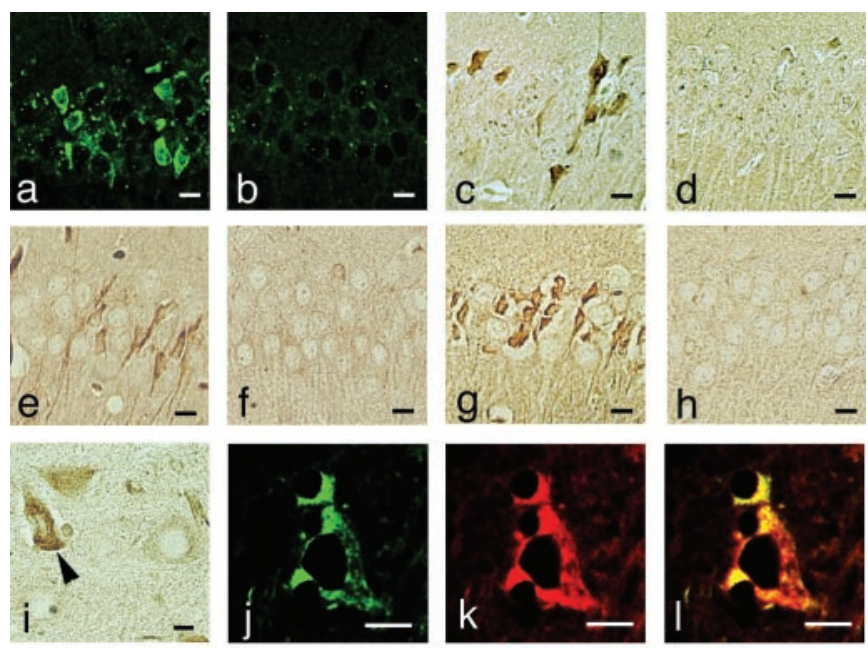

\section{h}
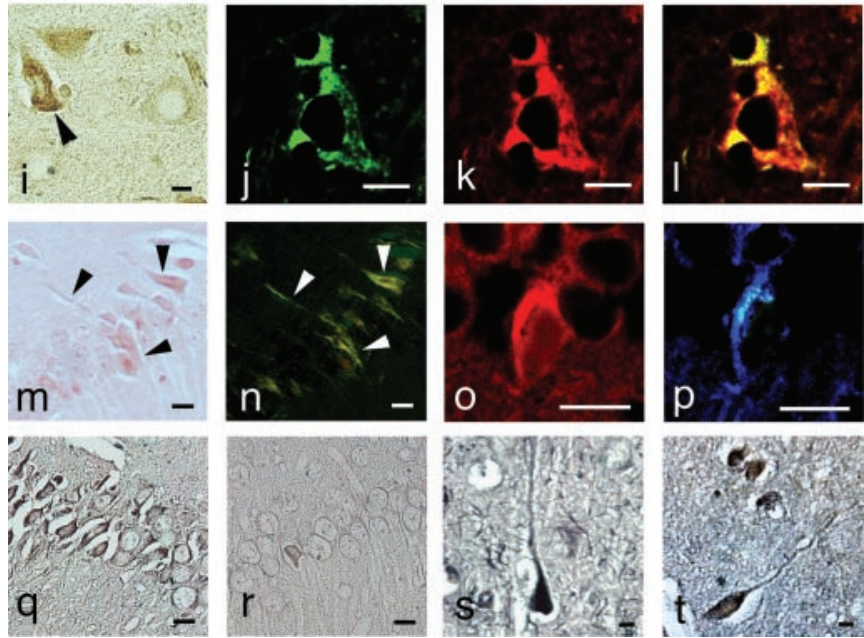

Fig. 2. Formation of tau inclusions in aged Tg mice. (a- $h$ ) Immunostaining of the hippocampal region (CA1) of a 19-month-old Tg mouse $(a, c, e$, and $g$ ) and a non-Tg littermate $(b, d, f$, and $h$ ) with monoclonal anti-myc antibody (a and $b$ ), PS199 ( $c$ and $d$ ), AT180 ( $e$ and $f$ ), and PS404 ( $g$ and $h$ ). (i) Immunostaining with Alz50. An arrowhead indicates a tangle-like inclusion in a large neuron of the striatum. $(j-l)$ Double immunostaining of the hilar neurons of the $\mathrm{Tg}$ hippocampus with JM $(k)$ and monoclonal anti-ubiquitin antibody $(j)$. A merged image is shown in $I$. ( $m$ and $n$ ) Photomicrographs of Congo redstained sections. The images were photographed without $(m)$ or with $(n)$ a polarizing filter set. Arrowheads indicate Congo red birefringent fibrils. (o and $p$ ) A neuron of the $\mathrm{Tg}$ hippocampus stained with polyclonal anti-tau antibody (JM) (o) was further stained with thioflavin $S(p) .(q-t)$ Photomicrographs showing Gallyas silver-stained sections of the hippocampus $(q)$, neocortex $(s)$, and amygdala $(t)$ from the Tg mouse and the hippocampus from the non-Tg littermate $(r)$. (Scale bars $=10 \mu \mathrm{m}$.)

To gain more direct insight into the effects of these changes on pathophysiology, we subjected $\mathrm{Tg}$ mice and their non-Tg littermates (16-23 months of age) to a comprehensive battery of behavioral tests assessing sensorimotor functions, emotional behaviors, and learning abilities. No significant differences were found between $\mathrm{Tg}$ mice and their non-Tg littermates regarding physical characteristics (body weight, temperature), or to sensorimotor reflexes (acoustic startle response), and motor coordination (accelerating rotarod and pole tests) (Table 1). We found a slight decrease in the locomotor activity of $\mathrm{Tg}$ mice during the first $5 \mathrm{~min}$ of the open field test, but overall evaluation using repeated-measures ANOVA revealed no significant difference. We found a slight but significant decrease in the prepulse inhibition test in Tg mice. Mortality of the Tg mice was higher than that of non-Tg littermates.

R406W Tg mice showed a significant impairment in the contextual and cued fear conditioning test (Fig. 5). Fear conditioning is a simple associative form of learning in which both a novel environment and a tone are paired with a foot shock on the training day. Memory is assessed by measurement of the amount of freezing (i.e., the fear response) elicited by either the same 
A a

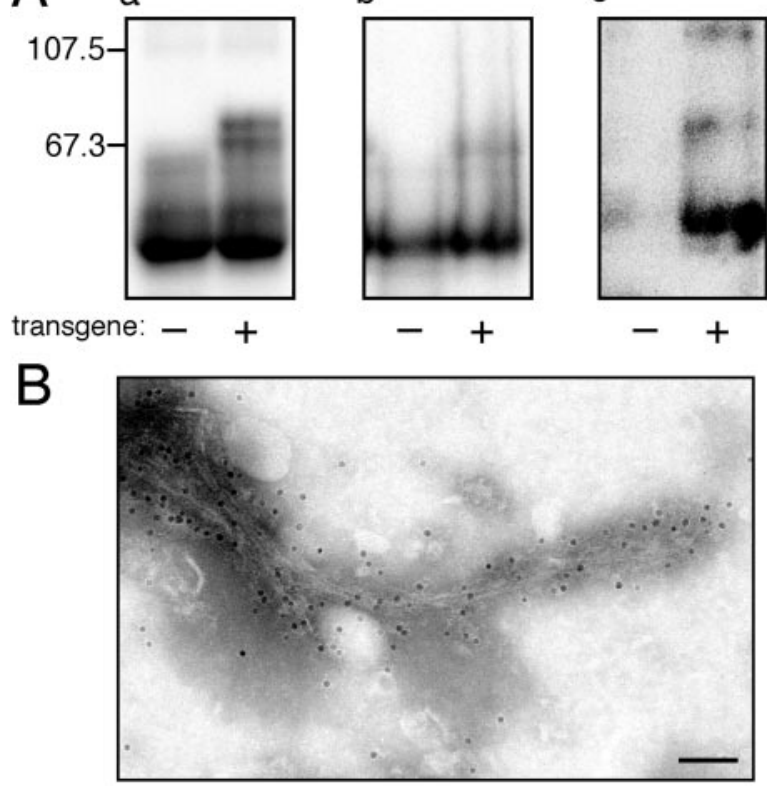

Fig. 3. Tau filament formation in aged Tg mice. (A) Western blot analysis of Sarkosyl-insoluble tau. TBS-soluble (a), Sarkosyl-soluble (b), and Sarkosylinsoluble $(c)$ fractions from 22-month-old control $(-)$ and $\mathrm{Tg}(+)$ brains were subjected to SDS/PAGE and stained with pan-tau antibody JM. (B) An immunoelectron photomicrograph of Sarkosyl-insoluble tau. Abnormal thin filaments intensely labeled by JM were recovered from Sarkosyl-insoluble fractions of the $\mathrm{Tg}$ brain but never from those of the control brain. (Scale bar $=50 \mu \mathrm{m}$.)

environment (contextual test, $24 \mathrm{~h}$ or 15 days later), or the tone (cued test, $48 \mathrm{~h}$ later), in the different environment. The contextual version of the task is selectively impaired by lesions
Table 1. Characteristic and sensorimotor functions of R406W Tg mice and their non-Tg littermates

\begin{tabular}{lcc} 
Measurement & Tg mice & Non-Tg mice \\
\hline Weight, g, 18 mo & $31.5 \pm 1.0$ & $29.3 \pm 0.6$ \\
Mortality, \% survived & & \\
18 mo & $90.9(20 / 22)$ & $100(21 / 21)$ \\
22 mo & $68.2(15 / 22)$ & $90.5(19 / 21)$ \\
Acoustic startle response, arbitrary unit, 23 mo & \\
Stimulus intensity = 70 dB & $10.3 \pm 0.7$ & $12.9 \pm 1.4$ \\
Stimulus intensity = 120 dB & $97.9 \pm 17.2$ & $123.8 \pm 4.3$ \\
Prepulse inhibition, \% (startle stimulus $=120 \mathrm{~dB}) 23 \mathrm{mo}$ & \\
Prepulse intensity = 70 dB & $13.2 \pm 7.9 *$ & $36.7 \pm 5.3$ \\
Prepulse intensity = 80 dB & $40.6 \pm 6.1$ & $55.7 \pm 4.3$ \\
Accelerating rotarod test, latency to fall, sec, $19 \mathrm{mo}$ & \\
Day 1 & $191 \pm 20$ & $161 \pm 22$ \\
Day 2 & $212 \pm 15$ & $198 \pm 24$ \\
Pole test, latency to fall, sec, 23 mo & $38.6 \pm 7.9$ & $36.8 \pm 6.6$ \\
Open-field test, horizontal activity, cm, $19 \mathrm{mo}$ & \\
0-5 min & $1,599 \pm 169$ & $2,168 \pm 239$ \\
5-10 min & $1,164 \pm 78$ & $1,338 \pm 155$ \\
$10-15$ min & $1,066 \pm 96$ & $972 \pm 127$ \\
15-20 min & $1,174 \pm 141$ & $1,123 \pm 82$ \\
20-25 min & $998 \pm 97$ & $888 \pm 105$ \\
25-30 min & $1,019 \pm 73$ & $820 \pm 89$ \\
\hline
\end{tabular}

$*, P<0.05$.

of the hippocampus, whereas both the cued and contextual versions of the task are impaired by lesions of the amygdala (29).

During the conditioning period, Tg mice showed significantly lower levels of freezing after foot shocks (conditioning; data not shown) [genotype effect, $F(1,15)=6.834 ; P=0.0195$ ]. The distance traveled just before ( $2 \mathrm{sec})$, during $(2 \mathrm{sec})$, and immediately after $(2 \mathrm{sec})$ the foot shocks was almost the same between
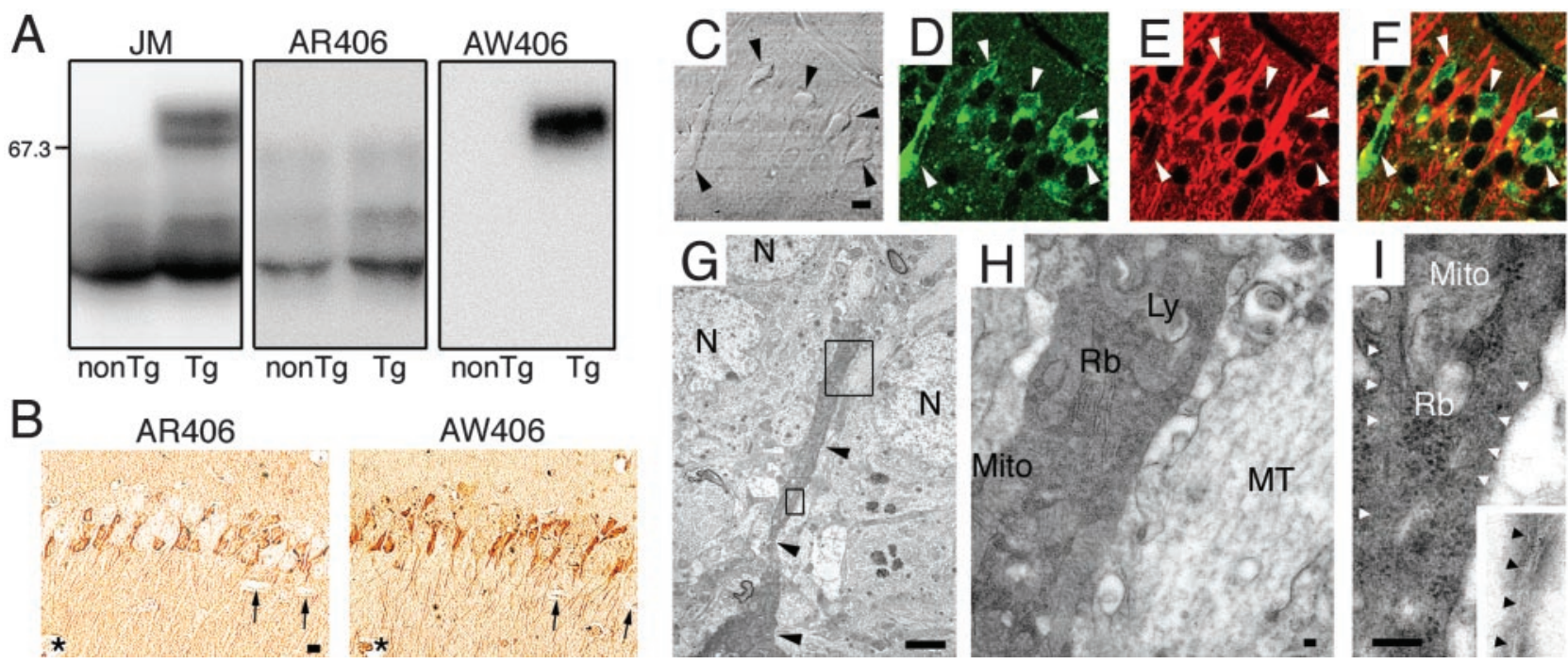

Fig. 4. Characterization of tau inclusions in aged Tg mice. (A) Western blots comparing the specificity of polyclonal antibodies AR406 and AW406 (19) with the anti-tau antibody JM. AR406 selectively stained mouse wild-type tau, whereas AW406 stained only mutant human tau. (B) Photomicrographs of two serial sections stained with AR406 and AW406. Note that both antibodies stained the same irregularly shaped neurons in the CA1 region of the aged Tg hippocampus. Arrows and asterisks indicate blood vessels. (C-F) Double immunostaining with AR406 and anti- $\alpha$-tubulin antibodies. Note that irregularly shaped neurons (arrowheads in C; phase contrast) in the aged Tg hippocampus are stained with AR406 (D) but lack tubulin immunoreactivity ( $E$ ). A merged image is shown in $F$. (Scale bars in $B$ and $C=10 \mu \mathrm{m}$.) (G-l) An ultrastructural analysis of an irregularly shaped neuron in the CA2 of aged Tg hippocampus. Arrowheads in $G$ indicate the somatodendritic part of an irregularly shaped neuron. N, nucleus. (Scale bar $=20 \mu \mathrm{m}$.) Boxed images in $G$ are enlarged in $H$ (large box) and $I$ (small box). Note that the dendrite of a degenerating neuron was electron-dense, filled with organelles $(H)$ and sometimes with bundles of thin filaments (white arrowheads in I). Negatively stained tau filaments (black arrowheads) from the Sarkosyl-insoluble fraction used in Fig. 3 were scale-adjusted and shown in the inset in I. Ly, lysosome; Rb, ribosome; Mito, mitochondria; MT, microtubules. (Scale bars in $H$ and $I=100 \mathrm{~nm}$.) 
a

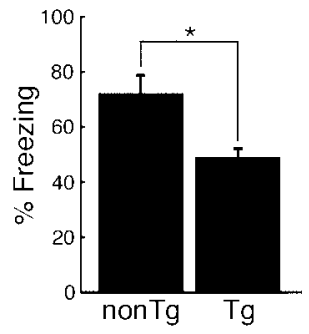

b

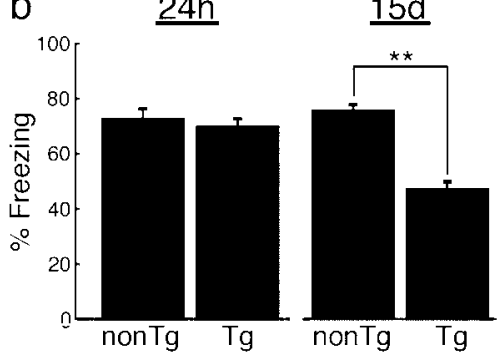

Fig. 5. Impaired associative memory in aged Tg mice. (a) Tg mice (19 months old) showed reduced levels of freezing during cued testing $(*, P=0.0359)$ conducted $48 \mathrm{~h}$ after conditioning. (b) Tg mice showed significantly reduced levels of freezing during contextual testing conducted 15 days after conditioning (15 d; **, $P=0.0072$ ). There was no significant difference in freezing between genotypes during contextual testing conducted $24 \mathrm{~h}$ after conditioning (24 h; $P=0.6291)$.

$\mathrm{Tg}$ and non-Tg mice (data not shown) [genotype effect, $F$ (1, $15)=1.648 ; P=0.2187]$. This finding excludes the possibility that reduced sensitivity to a foot shock is responsible for the reduced freezing levels in $\mathrm{Tg}$ mice. When the conditioned stimulus (tone) was presented in an altered context $48 \mathrm{~h}$ after conditioning (i.e., cued testing), Tg mice showed reduced levels of freezing [Fig. $5 a$, genotype effect, $F(1,15)=5.387 ; P=$ 0.0359]. Because no significant difference was found in the acoustic startle response or open-field tests, it is unlikely that this reduction was because of the reduced acoustic sensitivity or increased locomotor activity of the aged $\mathrm{Tg}$ mice.

During contextual testing conducted $24 \mathrm{~h}$ after conditioning, $\mathrm{Tg}$ mice showed the same levels of freezing as their non-Tg littermate controls [Fig. $5 b, 24 \mathrm{~h}$, genotype effect, $F(1,15)=$ $0.244 ; P=0.6291]$, suggesting that contextual fear memory developed both in the Tg and non-Tg mice. In the contextual testing conducted 15 days after conditioning, whereas controls showed freezing levels similar to those seen $24 \mathrm{~h}$ after conditioning, Tg mice displayed a significant reduction in freezing levels [Fig. 5b, 15d, genotype effect, $F(1,15)=10.447 ; P=$ $0.0072]$. This result suggests that memory loss may be more pronounced in longer retention delays in aged $\mathrm{Tg}$ mice.

\section{Discussion}

In the present study, we report the construction of $\mathrm{Tg}$ mouse lines expressing modest levels of R406W human tau confined predominantly to forebrain neurons. Importantly, these neurons develop argyrophilic and congophilic tau inclusions, and these $\mathrm{Tg}$ mice develop associative memory deficits. This finding suggests that modest levels of mutant (R406W) tau expression in neurons can cause neurodegenerative disease in mice. In contrast to previously reported $\mathrm{Tg}$ mice expressing wild-type or mutant human tau in both the brain and spinal cord, our mice expressed mutant tau mainly in forebrain neurons under the control of the CaMK-II promoter. This regional specificity enabled us to exclude with higher confidence possible confounding motor dysfunctions associated with neurogenic muscle atrophy (Table 1 and Fig. $5 b$ ). Because neurogenic motor symptoms can cause disruptions in memory performance in the behavioral tests that are unrelated to learning and memory function per se, our mice provide an animal model of CNS tauopathy for evaluating the relationships among mutant tau expression, neurofibrillary formation, and neuronal disturbance leading to memory impairment.

Congophilic tau inclusions developed only in a subset of forebrain neurons from aged Tg mice as early as 18 months of age. These inclusions fulfilled several criteria for the detection of human NFTs, including the ability to be stained by histochemical dyes that

bind crossed $\beta$-pleated sheet structures, such as Congo red and thioflavin $\mathrm{S}$, the ability to be impregnated by the Gallyas silver method, the presence of tau protein in isolated filaments from detergent-insoluble fractions, and the detection of ubiquitin immunoreactivity. Ishihara et al. (30) reported similar tau inclusions in forebrain neurons from aged (18-20 months) Tg mice expressing $3 \mathrm{R}$ wild-type human tau. However, the levels of human tau expressed in these mice were $\approx 5$-fold more than that of endogenous mouse tau. Given the modest $(\approx 20 \%)$ levels of mutant tau expression in our $\mathrm{Tg}$ mice, the development of tau inclusions in similar regions in similar ages was surprising. This finding might reflect one of the deleterious properties acquired by R406W mutation that promotes neurofibrillary formation, as in FTDP-17.

The major difference between the tau fibrils in our $\mathrm{Tg}$ mice and those in AD or R406W FTDP-17 is that tau filaments recovered from our aged $\mathrm{Tg}$ mice were mainly straight. This result may be because of altered tau isoform ratios or the combination of human and mouse tau in the filaments. In Tg mice, the three endogenous mouse wild-type (4R) and the longest human tau (4R) were expressed and incorporated into the inclusions, whereas in AD or R406W FTDP-17, all six tau isoforms (3R and 4R) were incorporated into the NFTs $(3,17)$. In contrast, we noted some similarities in tau fibril formation between our Tg mice and $\mathrm{AD}$ and R406W FTDP-17. For example, all of the tau isoforms expressed were incorporated into the tangles in proportion to their expression levels. This type of tangle formation appeared to be most common in many tauopathies in which altered proportion of tau isoforms directly reflected the tau isoform ratios incorporated into the tangles.

We have reported the behavioral but not memory-related abnormalities in V337M Tg mice (24). In the present study, we subjected aged (16-23 months) $\mathrm{Tg}$ mice and their non- $\mathrm{Tg}$ littermates to a comprehensive battery of behavioral tests and found impairment in associative memory retrieval without detectable sensorimotor deficits. Importantly, we found congophilic tau inclusions predominantly in the hippocampus, amygdala, and neocortex, areas well established to be involved in memory formation. However, further quantitative analysis is required to confirm the relationship between memory impairment and the formation of tau inclusions, because the appearance of tau inclusions in aged $\mathrm{Tg}$ mice is variable.

$\mathrm{Tg}$ mice showed reduced levels of fear response during the cued but not the contextual testing relatively soon after the initial conditioning (within $48 \mathrm{~h}$ ). Conditioning to a tone requires at least two different projections, one from the auditory cortex or thalamus to the lateral nucleus of the amygdala, and the other from the lateral nucleus to the central nucleus of the amygdala (29). Because the latter projection is also used for the contextual memory, the former projection might be disproportionately impaired in aged $\mathrm{Tg}$ mice. In addition, we found a significant decrease in the prepulse inhibition of the startle response, suggesting the impairment of inhibitory modulation by the auditory cortex.

$\mathrm{Tg}$ mice showed significantly lower levels of fear response during the contextual testing conducted 15 days after conditioning. Recently, a similar pattern of memory loss, i.e., impairment in the establishment of permanent memory, has been reported in mice heterozygous for a null mutation of CaMK-II (CaMK$\mathrm{II}^{+/-}$) (31). Because our Tg mice express mutant tau under the control of the CaMK-II promoter, it is plausible that the formation of tau inclusions and/or mutant tau expression itself might affect the CaMK-II-dependent establishment of permanent memory in $\mathrm{Tg}$ mice.

Our results represent a beginning in elucidating the molecular and cellular mechanisms underlying R406W tau mutation, which cause a tauopathy that clinically resembles AD. Because most of the R406W brains lack amyloid $\beta$ deposition (17), the adverse properties exhibited by this mutation might potentially recapitulate some early pathophysiological events in AD. Therefore, 
investigation of the molecular mechanisms of memory disturbance in our Tg mice could provide an important insight into the pathogenesis of both R406W FTDP-17 and AD.

We thank Dr. M. Mayford for providing the CaMK-II promoter. Y.T. thanks Prof. M. Takeda (Osaka University Graduate School of Medicine)

1. Reed, L. A., Wszolek, Z. K. \& Hutton, M. (2001) Neurobiol. Aging 22, 89-107.

2. Heutink, P. (2000) Hum. Mol. Genet. 9, 979-986.

3. Lee, V. M. \& Trojanowski, J. Q. (1999) Neuron 24, 507-510.

4. van Swieten, J. C., Stevens, M., Rosso, S. M., Rizzu, P., Joosse, M., de Koning, I., Kamphorst, W., Ravid, R., Spillantini, M. G., Niermeijer, P., et al. (1999) Ann. Neurol. 46, 617-626.

5. Spillantini, M. G. \& Goedert, M. (1998) Trends Neurosci. 21, 428-433.

6. Hutton, M., Lendon, C. L., Rizzu, P., Baker, M., Froelich, S., Houlden, H., Pickering-Brown, S., Chakraverty, S., Isaacs, A., Grover, A., et al. (1998) Nature 393, 702-705.

7. Poorkaj, P., Bird, T. D., Wijsman, E., Nemens, E., Garruto, R. M., Anderson, L., Andreadis, A., Wiederholt, W. C., Raskind, M. \& Schellenberg, G. D. (1998) Ann. Neurol. 43, 815-825.

8. Spillantini, M. G., Murrell, J. R., Goedert, M., Farlow, M. R., Klug, A. \& Ghetti, B. (1998) Proc. Natl. Acad. Sci. USA 95, 7737-7741.

9. Goedert, M., Spillantini, M. G., Crowther, R. A., Chen, S. G., Parchi, P., Tabaton, M., Lanska, D. J., Markesbery, W. R., Wilhelmsen, K. C., Dickson, D. W., et al. (1999) Nat. Med. 5, 454-457.

10. Grover, A., Houlden, H., Baker, M., Adamson, J., Lewis, J., Prihar, G., Pickering-Brown, S., Duff, K. \& Hutton, M. (1999) J. Biol. Chem. 274, 15134-15143.

11. Varani, L., Hasegawa, M., Spillantini, M. G., Smith, M. J., Murrell, J. R., Ghetti, B., Klug, A., Goedert, M. \& Varani, G. (1999) Proc. Natl. Acad. Sci. USA 96, 8229-8234.

12. Rizzu, P., Joosse, M., Ravid, R., Hoogeveen, A., Kamphorst, W., van Swieten, J. C., Willemsen, R. \& Heutink, P. (2000) Hum. Mol. Genet. 9, 3075-3082.

13. Miyasaka, T., Morishima-Kawashima, M., Ravid, R., Kamphorst, W., Nagashima, K. \& Ihara, Y. (2001) J. Neuropathol. Exp. Neurol. 60, 872-884.

14. Lewis, J., McGowan, E., Rockwood, J., Melrose, H., Nacharaju, P., Van Slegtenhorst, M., Gwinn-Hardy, K., Paul Murphy, M., Baker, M., Yu, X., et al. (2000) Nat. Genet. 25, 402-405.

15. Götz, J., Chen, F., Barmettler, R. \& Nitsch, R. M. (2001) J. Biol. Chem. 276, $529-534$. for the opportunity to participate in the project. This work is partly supported by Core Research for Evolutional Science and Technology (CREST), Japan Science and Technology (JST), a Grant-in-Aid for Scientific Research on Priority Areas (the Japanese Ministry of Education, Science, and Culture), and a Grant-in-Aid for Scientific Research (11680746, the Japanese Ministry of Education, Science, and Culture).

16. Spillantini, M. G., Crowther, R. A. \& Groedert, M. (1996) Acta Neuropathol. 92, 42-48.

17. Reed, L. A., Grabowski, T. J., Schmidt, M. L., Morris, J. C., Goate, A., Solodkin, A., Van Hoesen, G. W., Schelper, R. L., Talbot, C. J., Wragg, M. A. et al. (1997) Ann. Neurol. 42, 564-572.

18. Hong, M., Zhukareva, V., Vogelsberg-Ragaglia, V., Wszolek, Z., Reed, L., Miller, B. I., Geschwind, D. M., Bird, T. D., McKeel, D., Goate, A., et al. (1998) Science 282, 1914-1917.

19. Miyasaka, T., Morishima-Kawashima, M., Ravid, R., Heutink, P., van Swieten, J. C., Nagashima, K. \& Ihara, Y. (2001) Am. J. Pathol. 158, 373-379.

20. Lim, F., Hernandez, F., Lucas, J. J., Gomez-Ramos, P., Moran, M. A. \& Avila, J. (2001) Mol. Cell. Neurosci. 18, 702-714.

21. Mayford, M., Wang, J., Kandel, E. L. \& O'Dell, T. (1995) Cell 81, 891-904.

22. Burgin, K. E., Waxham, M. N., Rickling, S., Westgate, S. A., Mobley, W. C. \& Kelly, P. T. (1990) J. Neurosci. 10, 1788-1798.

23. Tanemura, K., Akagi, T., Murayama, M., Kikuchi, N., Murayama, O., Hashikawa, T., Yoshiike, Y., Park, J. M., Matsuda, K., Nakao, S., et al. (2001) Neurobiol. Dis. 8, 1036-1045.

24. Tanemura, K., Murayama, M., Akagi, T., Hashikawa, T., Tominaga, T., Ichikawa, M., Yamaguchi, H. \& Takashima, A. (2002) J. Neurosci. 22, 133-141.

25. Takashima, A., Murayama, M., Murayama, O., Kohno, T., Honda, T., Yasutake, K., Nihonmatsu, N., Mercken, M., Yamaguchi, H., Sugihara, S., et al. (1998) Proc. Natl. Acad. Sci. USA 95, 9637-9641.

26. Wolozin, B. L., Pruchnicki, A., Dickson, D. W. \& Davies, P. (1986) Science 232, $648-650$.

27. Greenberg, S. G. \& Davies, P. (1990) Proc. Natl. Acad. Sci. USA 87, 5827-5831.

28. Miyakawa, T., Yamada, M., Duttaroy, A. \& Wess, J. (2001) J. Neurosci. 21, 5239-5250.

29. LeDoux, J. E. (2000) Annu. Rev. Neurosci. 23, 155-184.

30. Ishihara, T., Zhang, B., Higuchi, M., Yoshiyama, Y., Trojanowski, J. Q. \& Lee, V. M. (2001) Am. J. Pathol. 158, 555-562.

31. Frankland, P. W., O’Brien, C., Ohno, M., Kirkwood, A. \& Silva, A. J. (2001) Nature 411, 309-313. 